Hadron Structure '15

International Journal of Modern Physics: Conference Series

Vol. 39 (2015) 1560091 (8 pages)

(C) The Author(s)

DOI: 10.1142/S2010194515600915

\title{
Latest Higgs physics results with the ATLAS detector
}

\author{
Domizia Orestano \\ (on behalf of the ATLAS Collaboration) \\ Dipartimento di Matematica e Fisica, Università Roma Tre and \\ INFN Sezione di Roma Tre, Via della Vasca Navale n.84 \\ Roma, 00143, Italy \\ orestano@fis.uniroma3.it
}

Published 26 November 2015

\begin{abstract}
This report presents the investigations on the recently discovered scalar boson by the ATLAS experiment at the CERN Large Hadron Collider. The latest results fully exploit the data collected during LHC Run 1 to measure the properties of the new boson and within the current sensitivity confirm the identification of this particle with the Higgs boson of the Standard Model.
\end{abstract}

Keywords: Higgs; boson; LHC.

PACS Numbers: 14.80.Bn, 13.85.-t

\section{Introduction}

The 4th of July 2012 the ATLAS ${ }^{1}$ and CMS $^{2}$ experiments, operating at the CERN Large Hadron Collider (LHC), announced the observation of a new boson with a mass close to $125 \mathrm{GeV}$. The discovery ${ }^{3,4}$ was based on about $5 \mathrm{fb}^{-1}$ collected at $\sqrt{s}=7 \mathrm{TeV}$ in 2011 and $5 \mathrm{fb}^{-1}$ collected at $\sqrt{s}=8 \mathrm{TeV}$ in 2012 by each experiment. At the time of the discovery the excess was significant in the two decay modes with the best mass resolution, $\gamma \gamma$ and $Z Z^{*}$, although there was already a clear indication also in the $W W^{*}$ channel. By the end of 2012 each of the experiments accumulated more than $\sim 20 \mathrm{fb}^{-1}$ of high quality data at $\sqrt{s}=8 \mathrm{TeV}$ which were used to consolidate this result, increasing the excess significance and the number of investigated channels thus shifting from discovery to measurements of the properties of the new particle.

This talk does not cover the details of the extraordinary performance of the accelerator and of the ATLAS experiment in 2012, nor the many technical and experimental issues, like the increasing pile-up of multiple collisions, which were

This is an Open Access article published by World Scientific Publishing Company. It is distributed under the terms of the Creative Commons Attribution 3.0 (CC-BY) License. Further distribution of this work is permitted, provided the original work is properly cited. 
successfully faced and overcome by the first analyses. It rather summarizes the most recent results of the ATLAS experiment from the final analysis of Run 1 data (collected in 2011 and 2012), focusing on the mature analyses developed during the Long Shutdown 1 of LHC (2013 and 2014) and comparing the properties of the new particle with Standard Model (SM) expectations for the Higgs boson.

\section{Production and Decay of the Standard Model Higgs Boson}

The Higgs boson couples both to fermions and to bosons and the couplings depend linearly on the fermionic masses and quadratically on the bosonic masses. These properties dictate the relative weight of the possible production modes at LHC. The most relevant ones are $^{5}$ :

- the gluon-gluon fusion (ggF) mechanism which, due to the lack of direct couplings of the Higgs boson to gluons, is mediated by a top-quark dominated loop, thanks to the mass dependence of the fermionic couplings. This process for $\sqrt{s}=8 \mathrm{TeV}$ has a cross section of $\sim 19 \mathrm{pb}$.

- the vector boson fusion (VBF) mechanism, which depends on the coupling of the Higgs boson to the $W$ and $Z$ bosons, with a cross section of $\sim 1.6 \mathrm{pb}$ at the $8 \mathrm{TeV}$ center-of-mass energy.

- the $V H$ associate production, where a weak boson $V=W$ or $Z$, produced by the electroweak interaction of a quark and an anti-quark, radiates a Higgs boson. The cross section at $8 \mathrm{TeV}$ is $\sim 1.1 \mathrm{pb}$.

- the associate production with a heavy $q \bar{q}$ pair, dominated by the heaviest pair, $t \bar{t}$, with a cross section of $0.13 \mathrm{pb}(t t H)$.

The Higgs boson then decays to pairs of massive weak bosons, or to pairs of fermions, or to not directly-coupled final states through fermionic or bosonic loops. The latter are dominated by top quark or $W$ bosons, which are the most massive objects of each category and can lead to gluon pairs (only through a top loop), $\gamma \gamma$ or $Z \gamma$ (with both contributions). The partial width of each decay channel depends on the mass of the involved particles, both through the coupling strength and the available phase space in the final state. The phase space contributions strongly depend on the Higgs boson mass which dictates the available decay channels. Therefore both the total width and the decay branching ratios (BR) depend strongly on the Higgs boson mass. For a $125 \mathrm{GeV}$ Higgs boson the main decay channels are $b \bar{b}$, with a BR of $\sim 58 \%$, and $W W^{*}$, with a BR of $\sim 22 \% .^{5}$ However these channels are not the easiest ones from an experimental point of view.

\section{Decay Channels}

Pros and cons of the main decay channels of the Higgs boson are illustrated in Table 1, in terms of BR, sensitivity to the different production modes, expected number of events, signal over background ratio $(\mathrm{S} / \mathrm{B})$ and mass resolution. 
Table 1. Comparison between the Higgs boson decay channels. Here $\ell=\mathrm{e}$ or $\mu$. A mass of $125 \mathrm{GeV}$ is assumed.

\begin{tabular}{cccccc}
\hline Decay & BR & $\begin{array}{c}\text { Production } \\
\text { Modes }\end{array}$ & $\begin{array}{c}\text { Run 1 } \\
\text { Expected Yield }\end{array}$ & S/B & $\begin{array}{c}\text { Mass } \\
\text { Resolution }\end{array}$ \\
\hline$\gamma \gamma$ & 0.0023 & ALL & $\sim 450$ & 0.03 & $1-2 \%$ \\
$Z Z^{*} \rightarrow 4 \ell$ & $0.026 \times(2 \times 0.034)^{2}$ & ALL & $\sim 20$ & 1.6 & $1-2 \%$ \\
$W W^{*} \rightarrow \ell \nu \ell \nu$ & $0.21 \times(2 \times 0.11)^{2}$ & ALL & $\sim 500$ & 0.15 & $20 \%$ \\
$\tau \tau$ & 0.063 & ALL & $\sim 300$ & $0.01-0.30$ & \\
$b \bar{b}$ & 0.58 & $V H, t t H$ & $\sim 400$ & $0.01-0.10$ & \\
\hline$Z \gamma$ & 0.0015 & ggF & & & \\
$\mu \mu$ & 0.00022 & ggF, VBF & & & \\
invisible & absent in SM & ggF $, \mathrm{VBF}, V H$ & & & \\
\hline
\end{tabular}

\section{Mass}

From Table 1 the best mass resolution is provided by the $Z Z^{*}$ to charged leptons and $\gamma \gamma$ decays. The lower statistics available in the former channel is compensated by its higher purity and both channels can be used to provide a precise measurement of the Higgs boson mass. After a careful calibration of photons, electrons and muons energy-scales, the mass of the Higgs boson is derived from a combined fit to the reconstructed invariant mass spectra of the decay channels $H \rightarrow Z Z^{*} \rightarrow 4 \ell$ and $H \rightarrow \gamma \gamma$ as described in Ref. 6: $m_{\mathrm{H}}=125.36 \pm 0.37$ (stat.) \pm 0.18 (syst.) GeV. These measurements have been combined with similar ones from the CMS Collaboration to obtain the LHC mass measurement: ${ }^{7} m_{\mathrm{H}}=125.09 \pm 0.21$ (stat.) \pm 0.11 (syst.) $\mathrm{GeV}$. The four measurements from the two channels and from the two experiments agree well with each other within less than two standard deviations.

\section{Width}

The expected width for a SM Higgs boson with a mass of $125 \mathrm{GeV}$ is only $\sim 4 \mathrm{MeV}$, which is three orders of magnitude below the experimental resolution of the channels discussed above. There is therefore no direct access to a width measurement and alternative approaches are needed to test its compatibility with the SM.

One approach is to set constraints on the off-shell Higgs boson signal strength, using $Z Z$ and $W W$ high mass final states, which can be combined with observed on-shell yields and then turned into limits on $\Gamma_{\mathrm{H}} / \Gamma_{\mathrm{H}}^{S M}$. This is done in Ref. 8 under the assumptions that the relevant Higgs boson couplings are independent of the energy scale of the Higgs boson production and that the same K-factors apply to signal and backgrounds. The result translates into an observed (expected) 95\% CL upper limit on the Higgs boson total width of 22.7 (33.0) $\mathrm{MeV}$.

The search for non SM decays or for deviations in the measured branching ratios with respect to SM is related to the width measurement. The search for invisible Higgs boson decays belongs to the former class of searches and it is performed either studying the recoil of $W$ or $Z$ bosons against an invisibly decaying hypothesized Higgs boson, ${ }^{9,10}$ or tagging the Higgs boson produced through VBF exploiting the 
two forward-jets signature. ${ }^{11}$ The latter analysis sets an upper limit at 95\% CL on the invisible branching ratio of a $125 \mathrm{GeV}$ Higgs boson at $29 \%$.

\section{Quantum Numbers}

In order to establish the spin and parity quantum numbers of the $125 \mathrm{GeV}$ boson, a number of hypotheses alternative to the $\mathrm{SM}$ assignment $\left(J^{P}=0^{+}\right)$are tested against the SM one constructing appropriate test statistics, allowing also for deviations in the couplings to fermions and vector bosons with respect to the SM expectation. ${ }^{12}$ All models alternative to the SM are excluded at more than $99.9 \%$ confidence level. Moreover the decays to $Z Z^{*}$ and $W W^{*}$, and then to $4 \ell$ and $\mu \nu e \nu$ respectively, allow to investigate the tensor structure of the $H V V$ interaction vertex which, under the spin-0 hypothesis, does not show any deviations from SM.

\section{Signal Strength}

For each investigated final state a signal strength parameter $\mu$ is defined as the ratio between the measured product of cross section times the BR and the expected SM one.

\subsection{Main decay channels}

In all the analyses different event categories are defined with a twofold purpose:

(i) enhance the sensitivity by separating classes of events with different signal over background ratios;

(ii) extract, whenever possible, information on the different production mechanisms, exploiting the categories defined under the different decay signatures.

Profile likelihood fits are then used to globally extract signal and nuisance parameters for each single channel ${ }^{13-17}$ and their combination. Of particular relevance are the evidence for the decay to $\tau \tau$ which is the first direct evidence of the Yukawa coupling to fermions ${ }^{16}$ and the first evidence for the VBF mechanism, achieved in the $W W^{*}$ channel. ${ }^{18}$

\subsection{Rare decays}

Searches have been performed also on decay channels for which, with the first $25 \mathrm{fb}^{-1}$, ATLAS is not expected to be sentive to the SM yield, like $h \rightarrow \mu \mu^{19}$ and $h \rightarrow Z \gamma,{ }^{20}$ setting mass dependent upper limits on the signal strength.

\section{3. ttH production mode}

The rare production mode $t t H$ is particularly interesting since it gives direct access to the largest Yukawa coupling, the one for the top quark. It has been searched for 
in three different final states:

- $H \rightarrow b \bar{b}$, where few hundred events are expected but $\mathrm{S} / \mathrm{B}$ is only few percent; ${ }^{21}$

- $H$ to multi-leptons (through $W W^{*}, \tau \tau$ and $Z Z^{*}$ decays), where $\mathrm{O}(10)$ events are expected but $\mathrm{S} / \mathrm{B} \sim 10 \% ;^{22}$

- $H \rightarrow \gamma \gamma$, where $\mathrm{O}(1)$ event is expected and $\mathrm{S} / \mathrm{B} \sim 20 \%$. $^{23}$

All three searches so far set upper limits at 95\% CL on $\mu$.

\subsection{Combined results}

Figure 1 summarizes the signal strength measured in each channel and the combined one. ${ }^{24}$ It is possible to extract the signal strength parameters for the different production modes from the combined fit, assuming SM branching ratios for the Higgs boson decay and profiting of the categorization of events. This results in the values summarized in Fig. 2.

Introducing scale factors parametrizing possible deviation from the SM couplings, it is possible to interpret the results within different scenarios ${ }^{25}$ and extract

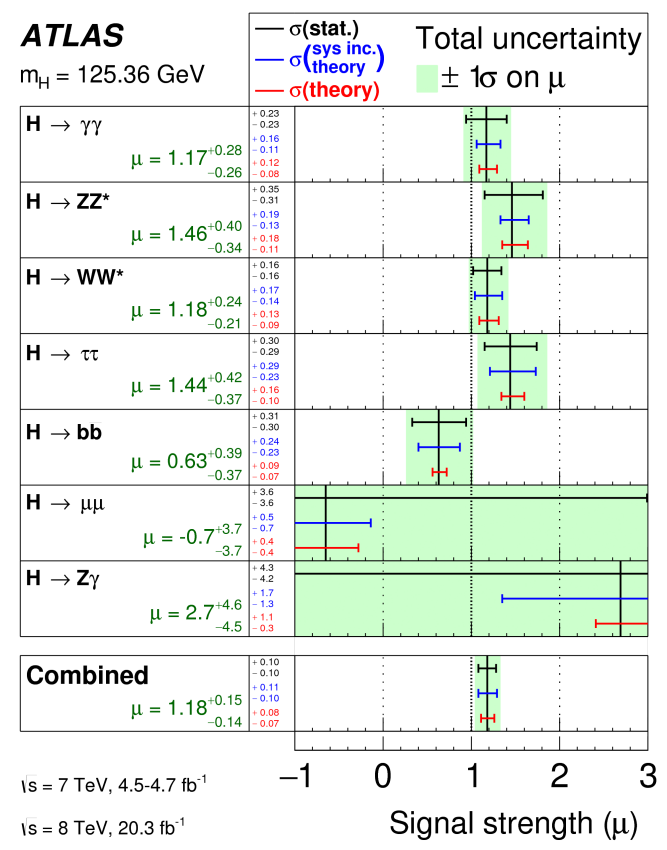

Fig. 1. The observed signal strengths and uncertainties for different Higgs boson decay channels and their combination for $m_{\mathrm{H}}=125.36 \mathrm{GeV}$. Higgs boson signals corresponding to the same decay channel are combined together for all analyses, assuming SM values for the cross-section ratios of different production processes. The best-fit values are shown by the solid vertical lines. The total $\pm 1 \sigma$ uncertainties are indicated by green shaded bands, with the individual contributions from the statistical uncertainty (top), the total (experimental and theoretical) systematic uncertainty (middle), and the signal theoretical uncertainty (bottom) on the signal strength shown as horizontal error bars. From Ref. 24. 


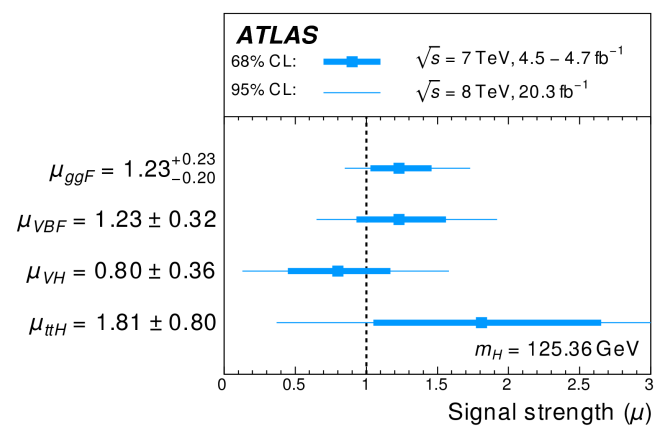

Fig. 2. The best-fit signal-strength values of different production modes determined from the combined fit to the $\sqrt{s}=7$ and $8 \mathrm{TeV}$ data. The inner and outer error bars correspond to $68 \%$ CL and 95\% CL intervals. Total uncertainties combining statistical, experimental and theoretical systematic uncertainties are shown. The fit assumes the SM values of the Higgs boson decay branching ratios for $m_{\mathrm{H}}=125.36 \mathrm{GeV}$. From Ref. 24 .

constraints on such models. The results presented in Ref. 24 appear well compatible with SM expectations.

\section{Cross Section}

The total and differential cross sections for the production of the Higgs boson in pp interactions have been measured from $\gamma \gamma$ and $Z Z^{*}$ candidates and are summarized in Ref. 26. The total cross sections measured in the two decay channels are perfectly compatible and their combined value is $\sigma=33.0 \pm 5.3$ (stat.) \pm 1.6 (syst.) pb. The systematic uncertainty includes the uncertainty on the Higgs boson mass, on the relative contributions of the different production modes, the acceptance variations due to the choice of parton density functions (PDF) and to the missing higher-order corrections, see Ref. 26 for more details. The central value of the ggF cross section, obtained subtracting a $3.0 \pm 0.1 \mathrm{pb}$ contribution for the other production modes,${ }^{5}$ is above the most recent theory SM predictions, although statistically in agreement, both for:

- next-to-next-to-leading order (NNLO) in QCD with threshold resummation accurate to next-to-next-to-leading logarithms (NNLL), see Ref. 5;

- and next-to-next-to-next-leading order ( $\left.\mathrm{N}^{3} \mathrm{LO}\right)$, see Refs. 27-30.

Differential cross sections are studied both to check for distortions possibly induced by new phenomena and to investigate if the mild excess observed in the total cross section is localized to specific kinematic regions. Differential distributions in inclusive and exclusive jet multiplicity bins, transverse momentum $\left(p_{\mathrm{T}}^{\mathrm{H}}\right)$ and rapidity $\left(y^{\mathrm{H}}\right)$ of the Higgs boson, and transverse momentum of the leading jet in the event $\left(p_{\mathrm{T}}^{\mathrm{j} 1}\right)$, are compared to various theoretical predictions and, within the still limited statistics, no deviations are observed. The $p$-values quantifying the compatibility between the measured cross sections and the predictions range from 

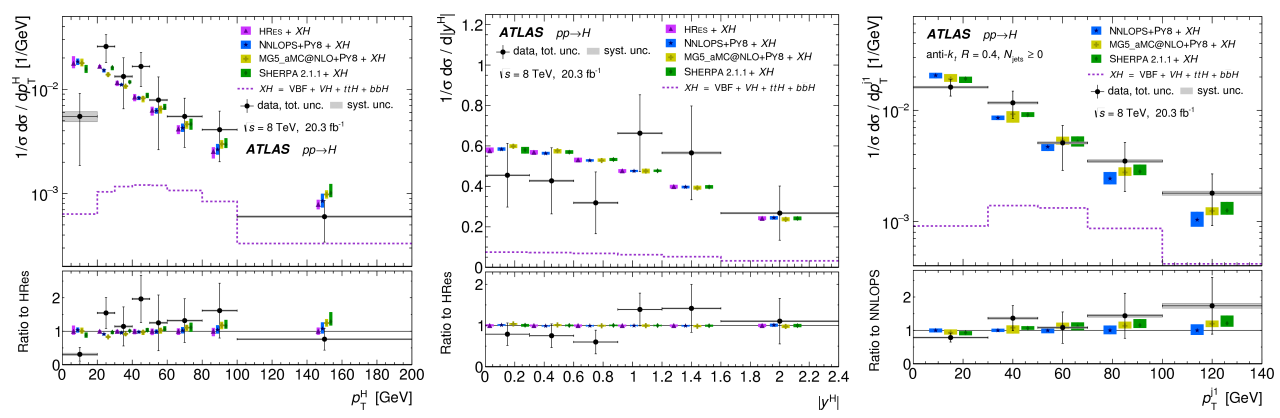

Fig. 3. Normalized cross-section shapes for inclusive Higgs boson production measured by combining the $H \rightarrow \gamma \gamma$ and $H \rightarrow Z Z^{*} \rightarrow 4 \ell$ channels. The measured variables are, from left to right, the Higgs boson transverse momentum $p_{\mathrm{T}}^{\mathrm{H}}$ and its rapidity $y^{\mathrm{H}}$, and the transverse momentum of the leading jet $p_{\mathrm{T}}^{\mathrm{j} 1}$. The $0-30 \mathrm{GeV}$ bin of the $p_{\mathrm{T}}^{\mathrm{j} 1}$ distribution corresponds to events without jets above $30 \mathrm{GeV}$. Various ${ }^{31-37}$ theoretical predictions are presented, using the same bin widths as the measurement. Events simulated with NNLOPS and MG5_aMCNLO are interfaced with PYTHIA $8^{38}$ for parton showering. From Ref. 26.

$2 \%$ to $26 \%$, and for the shapes from $8 \%$ to $88 \%$. Figure 3 presents the measured cross-section shapes compared to predictions normalized to the data.

\section{Summary and Outlook}

Progress in Higgs boson physics did not stop over the Long Shutdown 1 of LHC. Significative steps were made in refining the analyses which led to the discovery of the new particle and the first tests of its characteristics. The $\sim 25 \mathrm{fb}^{-1}$ of data collected in Run 1 have been fully exploited and ATLAS is now ready to investigate the Higgs boson properties at the higher energies of Run 2. In the new Run, just started, ATLAS expects to collect ten times more integrated luminosity than in Run 1. The increased center-of-mass energy will push up the different Higgs boson production cross sections by factors ranging between 2.1 and 2.6 for the majority of the production channels and reaching 4.7 for $t t H$. Top quark related backgrounds will scale up by a similar factor.

\section{References}

1. ATLAS Collaboration, JINST 3, S08003 (2008).

2. CMS Collaboration, JINST 3, S08004 (2008).

3. ATLAS Collaboration, Phys. Lett. B716, 1 (2012).

4. CMS Collaboration, Phys. Lett. B716, 30 (2012).

5. LHC Higgs Cross Section Working Group, S. Dittmaier, C. Mariotti, G. Passarino and R. Tanaka (Eds.), Handbook of LHC Higgs Cross Sections: 1. Inclusive Observables, arXiv:1101.0593 (2011).

6. ATLAS Collaboration, Phys. Rev. D90, p. 052004 (2014).

7. ATLAS and CMS Collaborations, Phys. Rev. Lett. 114, 191803 (2015).

8. ATLAS Collaboration, Eur. Phys. J. C75, 335 (2015).

9. ATLAS Collaboration, Eur. Phys. J. C75, 337 (2015). 
10. ATLAS Collaboration, Phys. Rev. Lett. 112, 201802 (2014).

11. ATLAS Collaboration, Search for an Invisibly Decaying Higgs Boson Produced via Vector Boson Fusion in pp Collisions at $\sqrt{s}=8 \mathrm{TeV}$ using the ATLAS Detector at the LHC, ATLAS-CONF-2015-004 (Mar, 2015), http://cds.cern.ch/record/2002121.

12. ATLAS Collaboration, Study of the Spin and Parity of the Higgs Boson in Diboson Decays with the ATLAS Detector, arXiv:1506.05669 (2015).

13. ATLAS Collaboration, Phys. Rev. D 90, 112015 (2014).

14. ATLAS Collaboration, Phys. Rev. D 91, 012006 (2015).

15. ATLAS Collaboration, JHEP 08, 137 (2015).

16. ATLAS Collaboration, JHEP 04, 117 (2015).

17. ATLAS Collaboration, JHEP 01, 069 (2015).

18. ATLAS Collaboration, Phys. Rev. D 92, 012006 (2015).

19. ATLAS Collaboration, Phys. Lett. B 738, 68 (2014).

20. ATLAS Collaboration, Phys. Lett. B 732, 8 (2014).

21. ATLAS Collaboration, Eur. Phys. J. C 75, 349 (2015).

22. ATLAS Collaboration, Phys. Lett. B 749, 519 (2015).

23. ATLAS Collaboration, Phys. Lett. B 740, 222 (2015).

24. ATLAS Collaboration, Measurements of the Higgs Boson Production and Decay Rates and Coupling Strengths Using pp Collision Data at $\sqrt{s}=7$ and 8 TeV in the ATLAS Experiment, arXiv:1507.04548 (2015).

25. LHC Higgs Cross Section Working Group, S. Heinemeyer, C. Mariotti, G. Passarino and R. Tanaka (Eds.), Handbook of LHC Higgs Cross Sections: 3. Higgs Properties, arXiv:1307.1347 (2013).

26. ATLAS Collaboration, Phys. Rev. Lett. 115, 091801 (2015).

27. C. Anastasiou, C. Duhr, F. Dulat, E. Furlan, T. Gehrmann, F. Herzog and B. Mistlberger, Phys. Lett. B 737, 325 (2014).

28. C. Anastasiou, S. Buehler, F. Herzog and A. Lazopoulos, JHEP 12, 058 (2011).

29. C. Anastasiou, C. Duhr, F. Dulat, E. Furlan, T. Gehrmann, F. Herzog and B. Mistlberger, JHEP 03, 091 (2015).

30. C. Anastasiou, C. Duhr, F. Dulat, F. Herzog and B. Mistlberger, Phys. Rev. Lett. 114, 212001 (2015).

31. D. de Florian, G. Ferrera, M. Grazzini and D. Tommasini, JHEP 06, 132 (2012).

32. M. Grazzini and H. Sargsyan, JHEP 09, 129 (2013).

33. K. Hamilton, P. Nason, E. Re and G. Zanderighi, JHEP 10, 222 (2013).

34. R. Frederix and S. Frixione, JHEP 12, 061 (2012).

35. J. Alwall, R. Frederix, S. Frixione, V. Hirschi, F. Maltoni, O. Mattelaer, H. S. Shao, T. Stelzer, P. Torrielli and M. Zaro, JHEP 07, 079 (2014).

36. T. Gleisberg, S. Hoeche, F. Krauss, M. Schonherr, S. Schumann, F. Siegert and J. Winter, JHEP 02, 007 (2009).

37. S. Hoeche, F. Krauss and M. Schonherr, Phys. Rev. D90, 014012 (2014).

38. T. Sjostrand, S. Mrenna and P. Z. Skands, Comput. Phys. Commun. 178, 852 (2008). 